\title{
OBJETS DE PARURE DE LA NÉCROPOLE DE VADU ANEI
}

\author{
Luminița Dumitriu
}

\section{SCHMUCKSTÜCKE AUS DEM GRÄBERFELD VON VADU ANEI}

\section{Zusammenfassung}

Die Schmuck- und Trachtstücke aus dem Gräberfeld von Vadu Anei sind durch Ohrringe mit Kügelchen, mit Räderchen und mit kugekförmigem Anhänger, durch Perlen, einfache Fingerringe, einteilige Fingerringe mit Schild oder Fingerringe mit angelötetem Schild vertreten. Dazu wurden, als Kleidungszubehör, auch kugelförmige Knöpfe behandelt.

Die Analyse der 59 Schmuck- und Trachtstücke gestattet ihreZuweisung einer dörflichen Gemeinschaft vom Ausgang des XV, XVI, und auch vom Anfang des XVII Jahrhunderts.

\section{LA TYPOLOGIE DES OBJETS DE PARURE}

Le spectre typologique des pièces de parure et de port découvert dans la nécropole de Vadu Anei est représenté par des pièces de parure pour la tête, le cou, les mains et des accessoires vestimentaire

\section{Parures pour la tête.}

La seule catégorie trouvée dans le cadre de l'inventaire funéraire de Vadu Anei est représentée par les boucles d'oreille. En fonction de la manière de réalisation, forme et décor, ceux-ci peuvent être groupés dans plusieurs types.

\section{Boucles d'oreille.}

\subsection{Boucles d'oreille avec des granules}

Les boucles d'oreille travaillées de fil d'argent ou bronze (M.76, M. 313) contenant un anneau circulaire, ayant à la partie inférieure une granule en forme de bouton de fleur, obtenue par l'entrelacement de trois fils d'argent ou de bronze, chacun composé à son tour par la torsion d'autres deux fils, d'une part et de l'autre de la granule en forme de bouton, l'anneau de la boucle d'oreille étant entrelacée avec le même type de fil tordu. Seulement dans le cas des boucles d'oreille.en bronze de $\mathrm{M}$. 313, le fil est simple (Pl. 1/ 1-2).
Ce type de parure, un peu illustré dans la variété des boucles d'oreille appartenant aux $\mathrm{XIV}-\mathrm{e}-\mathrm{XV}$-e siècles, parait avoir son origine dans le type de boucles d'oreille avec bouton pétaliphorme de facture byzantino-balcanique (Dumitriu 2001, 38). Pour le XVI-e siècle le type apparaît documenté dans la nécropole de Piua Petri (Chițescu ş. a. 1982, 155, 145 fig. 10/ 21.).

1.2. Boucles d'oreille avec des petites roues granulées.

Les boucles d'oreille avec des petites roues granulées apparaissent en deux variantes

1.2a. Boucles d'oreille avec de petites roues granulées disposées en cercle.

Boucles d'oreille en argent ayant l'anneau demi-circulaire ornementés avec une pastille centrale, fixée à la partie inférieure, réalisée par la soudure de trois petites roues, qui ont le bord marqué par un suite de petits boutons granulés. D'une part et de l'autre de cet ornement, l'anneau apparaît tordu avec un fil d'argent filigrané. A l'un des bouts se trouve l'anneau de fixation, et à l'autre une pastille de petites dimensions, avec la même enfilade de granules disposée sur les côtes. Elles sont documentées dans M. 59 (deux exemplaires d'argent, Pl. 1/ 3) et sans contexte funéraire, par un fragment de bronze découvert dans la section XXXII, $\square 12$ (Pl. 1/ 4). 
1.2b. Boucles d'oreille avec de petites roues granulées disposées en pyramide.

Une autre variante de ce type est représenté par l'exemplaire découvert dans $\mathrm{M}$. 349 , chez le quel la petite roue centrale a les bords décorés avec des granules disposées en pyramide (Pl. 1/ 5).

Ce type de parure apparait souvent dans des nécropoles du XV-e siècle (Dumitriu 2001, 41 , avec la bibliographie). Il continue d'être utilisé aussi au XVI-e siècle, étant attesté dans les nécropoles de Bucarest - Bârzeşti (Panait, Ştefănescu 1992, 156 pl. 2) mais aussi à Piua Petri (Chițescu ş. a. 1979, 228, 233 fig. 18/ 8) Les dimensions réduites et la finesse de l'anneau inclus aussi ce type dans la catégorie des boucles d'oreilles proprement dites, qui étaient fixées dans le lobe de l'oreille.

\section{I.3. Boucles d'oreille à bouton sphéro- ïdal et des tubes cylindriques.}

La boucle d'oreille fragmentaire d'une feuille de métal commun, dorée à l'extérieure, d'une forme sphéroïdale de M. 246 (Pl. I/6), à été prévue dans la partie supérieure avec deux tubes cylindriques, par les quels passait l'anneau, et le corps décoré en gravure avec cinq registres d'ornement à rosettes. Le type pourrait provenir de la catégorie des boucles d'oreille qui apparaissent au XV-e siècle - les ainsi dites boucles d'oreille en forme de cadenas, probablement aussi elles d'origine balkanique (Dumitriu 2001, 40 - 41; Rosetti 1972, 32 pl. V/ 1, 33 pl. VI/ 8).

\section{Parures pour le cou.}

Les parures pour le cou sont représentées seulement par des enfilades de perles ou par des pièces singulières. Elles ont été faites pour leur grande partie en verre, de différentes couleurs (blanc, vert, gris, bleu, vert foncé, noire) et elles sont rectangulaires ou carrées en section (M.65, M. 72). Dans le cas de la sépulture M. 73, les perles découvertes, différentes comme forme, alternent avec celles circulaires (3 exemplaires), tubulaires (1 exemplaire) ou avec les rebords lobés ayant la forme d'une fleure à six pétales (1 exemplaire). On a découvert aussi des enfilades de perles de divers dimensions, facettées ou tout droites, quelques-unes étant ornementées avec des motifs géométriques, des lignes horizontales de couleur verte, rouge, bleu sur un fond gris vert (M. 207, M. 246). Un exemplaire à part a été découvert dans M. 425, représenté par une petite perle bitronconique, décorée avec des incisions de couleur blanche, sur un fond noir bleuâtre, motif semblable a une feuille de fougère.

La catégorie des perles est le plus souvent rencontrée dans les nécropoles médiévales, elles représentant peut être la plus meilleure marché et la plus accessible parure féminine (Dumitriu 2001, 43 - 46).

\section{Parures pour les mains (anneaux et bagues).}

Dans la nécropole de Vadu Anei les parures pour les mains, représentaient seulement par des anneaux et des bagues sont la catégorie la plus riche, non seulement par le grand nombre d'exemplaires (32 pièces), mais aussi par une appréciable variété typologique. A l'exception des anneaux de M.99 et de celui de M.445, faits en bronze, tous les autres exemplaires des contextes funéraires sont en argent. Les anneaux et les bagues sont représentés par les suivants types.

\subsection{Anneaux simples.}

Le type se rencontre sous deux variantes.

3.1a. Anneaux simples ayant le corps circulaire en section et les bouts tout proches.

La variante est représenté par un exemplaire en bronze (M. 99) (Pl. II/ 2) et deux en argent (M. 346, M. 421) (Pl. II/ 3 - 4) et encore un autre exemplaire en argent, ayant les bouts entre passés (M. 282) (Pl. II/ 5). De même a ce type appartient aussi un anneau en bronze, sans contexte funéraire, découvert dans la section XVI, $\square 5$ (Pl. II/ 1).

\section{1b. Anneaux simples ayant le corps} d'un fil en argent tordu.

Dans la nécropole de Vadu Anei on a découvert un nombre de neuf exemplaires: M.35, 46, 60, 62, 69, 153, 360, 381, 408 (Pl. II/ 6 - 14).

Ce type d'anneau, parfois utilisé aussi comme anneau de boucle, surtout aux X-e XII-e siècles, est souvent rencontré dans le répertoire funéraire en commencent avec le XIe siècle et jusqu'au XVIII-e siècle, étant l'objet de parure le plus facilement a faire, accessible 
pour toutes les catégories sociales (Dumitriu 2001, 30, 52)

\subsection{Bagues ayant l'anneau corps} commun avec la monture.

\section{Le type est représenté par deux} variantes. forme.

3.2a. Bagues ayant l'anneau bande-

La bague de la sépulture M.43 (Pl. III/ 1) en a la monture un peu surélevée et décorée à l'aide de la technique de l'incision avec un motif géométrique qui suggère la lettre „X”. La bague de M.45 (Pl. III/ 2), peut être une alliance de fiançailles, en a deux impressions niellées à sa partie supérieure mais aussi sur les épaules de l'anneau, légèrement en reliefs, exemplaire sans analogies connues dans le milieu funéraire médiévale villageois. circulaire.

3.2b. Bagues ayant l'anneau demi-

Les bagues ayant l'anneau demicirculaire sont les plus beaux exemplaires de l'inventaire funéraire de Vadu Anei. Si elles auraient été découvertes dans des autres contextes, par exemple dans des nécropoles de boyards ou urbaines, elles auraient pu être homologuées comme bagues sigillaires. Le milieu villageois, dans le quel s'encadre la nécropole de Vadu Anei, tenant compte aussi de l'absence d'un lieu de culte ou d'une éventuelle cour de boyard, la présentation de leur dimensions se résume seulement à leur caractère artistique. Ces bagues sont des pièces massifs, réalisées par moulage et ornées dans la technique de la gravure et parfois du niellage, comme par exemple la bague de M.310 (Pl. III/ 8).

La bague de la sépulture 98 (Pl. III/ 3) a été faite en argent doré, ayant une monture octogonale, ornementée avec des motifs géométriques gravés, représentant probablement le motif zodiacal du poisson, et les épaules de l'anneau sont gravées avec des motifs géométriques.

Les exemplaires des sépultures 335 (Pl. III/ 4) et 327 (Pl. VI), ont la monture décorée par incision avec des motifs solaires, de plus les épaules de l'anneau de la bague de M.335 sont décorées avec trois registres de lignes incisées. Les bagues de M.248 (Pl. III/ 6) et 341 (Pl. III/
5) ont été ornementées avec des motifs zoomorphes: une chèvre respectivement deux oiseaux en profil. La pièce de la sépulture $\mathrm{M}$. 63 (Pl. III/ 7) est la seule qui en a encastrée dans la monture surélevée, avec le bord ciselé, une pierre demi-précieuse, probablement un agate.

La bague de la sépultures 310 (Pl. III/ 8) peut être considérée, à cause de la monture décorée par gravure et niellage avec le motif de l'aigle bicéphale et de la fleur de double lys, comme le plus précieux exemplaire découvert à Vadu Anei, fait lui aussi, comme de même les autres exemplaires, dans des ateliers d'orfèvres urbains ou peut être même des imports du monde sud-danubien. Le motif de l'aigle bicéphale, motif ornementale d'origine byzantine a été documenté aussi dans la nécropole de MăneştiBuftea, sur une bague en argent ayant le chaton discoïdale, mais datant du XIV-e siècle (Ştefănescu 1979, 28 fig. e; Ştefănescu 1980, 139).

\subsection{Bagues à chaton soudé sur} l'anneau.

Dans la nécropole de Vadu Anei le type est représenté par deux variantes

3.3a. Bagues ayant l'anneau demicirculaire et le chaton rectangulaire.

A cette variante appartient seulement la bague de la sépulture 61 (Pl. IV/ 1), qui a le chaton rectangulaire, décoré avec la croix de Malte et avec les bras encadrés avec des carreaux en hachures.

3.3b. Bagues ayant l'anneau tordu et le chaton circulaire ou rhomboïdale.

Aux bagues des sépultures 3, 125, 126 et 344 les chatons sont circulaire-ovales. A la bague de la sépulture 125 (Pl. IV/ 3) la décoration du chaton est faite d'une rosette. Et à la bague de M.3 (Pl. IV/ 2) est représenté probablement le signe zodiacal du poisson. Aux autres deux bagues (Pl. IV/4-5), à cause d'une longue utilisation, le possible décor s'est effacé.

Le chaton des bagues des sépultures 11 (Pl. IV/ 6), 64 (Pl. IV/ 7), 202 (Pl. IV/ 8), et 233 (Pl. IV/ 9) est rhomboïdale et ornementé par incisions avec des motifs cruciformes ou florales, peut être une croix de Malta dans le cas de la sépulture 233 . 
Les bagues de ce type commencent a être utilisées vers la fin du XV-e siècle, pour qu'en suite elles deviennent des pièces fréquentes dans les inventaires funéraires des nécropoles villageoises des XV-e - XVII-e siècles (Panait, Ştefănescu 1992, 155, pl. I, Chițescu ş. a. 1981, 131 fig. 7/ 3-4)

\section{Accessoires vestimentaires.}

\subsection{Boutons.}

Les boutons, comme d'ailleurs aussi les perles, sont le plus souvent identifiés dans l'inventaire funéraire des nécropoles médiévales. A Vadul Anei ont été réalisés en deux hémisphères d'une feuille de métal commun, soudée dans la zone du diamètre maxime, ayant un anneau de fixage. Parfois ils ont été argentés (M.76, 253, 310, 379) et même dorés (M.21，47, 59, 100, 408, 421) à 1'extérieure. Souvent apparaissent aussi des exemplaires en argent (M.35, 51, 202, 360). Ils sont des pièces communes, non-décorés. Leur structure formative permet la distinction de deux sous-types. Beaucoup de fois l'état de conservation n'a pas permis l'identification exacte de la forme et des détailles typologiques

4.1a. Boutons globulaires ayant la partie inférieure biseautée $(\mathrm{Pl}$. V/1-2).

A ce sous type appartiennent par exemple les boutons de M.21, 35, 59, 100, 313, 360,421

4.1b. Boutons globulaires avec des protubérances à la partie inférieure $(\mathrm{Pl}$. V/34).

Boutons globulaires avec des protubérances documentés dans les sépultures 47 şi 408.

A cause de motifs objectifs la recherche de la nécropole de Vadu Anei n'a pas réussi d'identifier ni l'établissement ni le bâtiment de culte affairent. L'existence de celui-ci peut être supposée. Il est possible que lui n'a pas permit une extension non contrôlée de l'espace funéraire à cause des nombreux horizons de sépultures.

Manquant une analyse anthropologique complète, beaucoup de fois la détermination de la relation d'entre les pièces de parure et le sexe du porteur est aléatoire et préjudice l'image finale concernant les éléments de costume.
Mais pourtant on peut apercevoir quelques observations suggestives (fig. 1). Très intéressant est le fait que dans des sépultures d'enfant ont apparue, plus souvent, des boucles d'oreille, des perles et des boutons.

En ce qui concerne les boucles d'oreille, portés au temps par des adultes que par des enfants, elles n'apparaissent pas en association avec des bagues et elles n'existent pas dans les sépultures de mâles.

Dans le sépultures de femmes, autant que la recherche anthropologique a été faite, l'inventaire se compose seulement de trois bagues, boutons et probablement des perles.

Les sépultures de mâles sont les mieux illustrées par la présence de 25 bagues des 32 de exemplaires et qui remplissent toute la gamme typologique. Seulement dans une seule sépulture de mâle (M. 130) il y a aussi une perle.

Les boutons sont des accessoires vestimentaires commun pour toutes le sépultures.

L'analyse des pièces de parure de la nécropole de Vadu Anei permet sa datation à la fin du XV-e siècle, au cours du XVI-e et peut être au commencement du XVII-e siècle.

Même si seulement avec approximation $13 \%$ des sépultures contiennent des pièces de parure et des accessoires vestimentaires, on peu pourtant identifié une certaine stratification sociale, visible par les sépultures avec des bagues ayant l'anneau demi-circulaire et le corps commun avec la monture, pièces, parfois d'une impressionnante qualité artistique, qui dans d'autres milieux sont liées à une haute catégorie sociale.

En fin de compte on peu dire que les éléments obtenus de l'analyse de l'inventaire funéraire de la nécropole de Vadu Anei permettent l'enrichissement de l'image concernant les réalités historiques du milieu rural du XVI-e siècle, suggérant un niveau d'habitat relativement varié et un goût artistique de la même mesure, adapté aux possibilités matérielles propre pour la communauté rurale de Vadu Anei, qui se trouvait dans une étroite liaison avec le milieu urbain de Bucarest. 


\section{LE CATALOGUE DES PARURES}

M.3: Bague en argent ayant l'anneau en fil retors, sur le quel on a soudé comme monture une pastille circulaire. Le motif décoratif, réalisé par incision représente un signe zodiacal (le poisson). La pièce a l'anneau un peu déformé et la monture

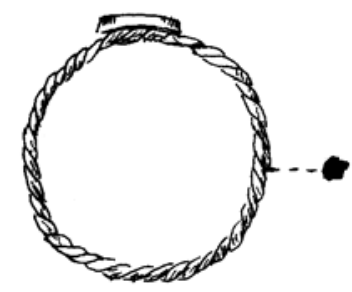
usée.

Dm.ext. anneau: 25mm; dm. monture: $9 \mathrm{~mm}$; $\mathrm{G} .: 3,87 \mathrm{gr}$.

M.11: Bague en argent ayant l'anneau tordu, sur le quel a été soudé une monture en forme de rhombe. Le décor incisé redonne le motif de la croix, avec les bras égaux, tout dans un cadre de lignes incisées.

Dm.ext. anneau: 25 $\mathrm{mm}$; côte de rhombe:

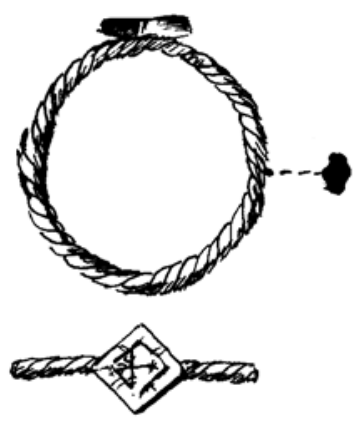

7 mm; G.: 3 gr.

M.12: Quatre petites anses en bronze de quatre boutons.

M.21: Deux boutons globulaires fragmentaires, réalisés d'une feuille de métal commun, dorés à l'extérieure, ayant la partie inférieure biseautée. Manque l'anneau de fixation.

Dm.: 4 mm.

M. 35: 1. Anneau d'argent d'un fil retors, ayant les bouts tout proches.

Dm.ext. anneau: $24 \mathrm{~mm}$; G.: 1,48 gr.

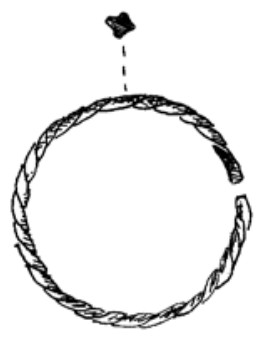

2. Quatre boutons (deux étant fragmentaires), faits

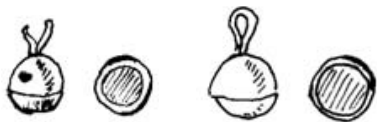

d'une feuille d'argent, d'une forme circulaire (deux hémisphères soudées), la partie inférieure biseautée.

Dm.ext. anneau entre 6-7 mm; h.: $11 \mathrm{~mm}$.

M. 36: Petites anses en bronze de trois boutons.

M.: 43: Bague d'argent avec l'anneau bandeforme, qui fait corps commun avec la monture. Celle-ci, d'une forme rectangulaire et un peu surélevée a comme décor deux lignes incisées ayant la forme de la lettre „X”. Les bords et

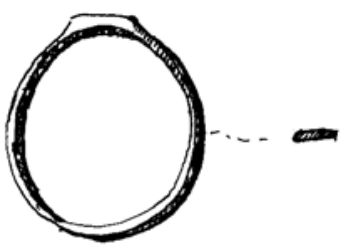
les épaules de la monture présentent des registres de lignes incisées. A la partie inférieure de l'anneau se garde encore les traces de la soudure.

Dm.ext. anneau: $20 \mathrm{~mm}$; monture: 7 x $6 \mathrm{~mm}$; G.: 2,55 gr.

M. 45: Bague d'argent avec l'anneau bandforme, qui fait corps commun avec la monture. L'anneau un peu envasé et défaite à la partie de la monture. Le décor réalisé à l'aide de deux

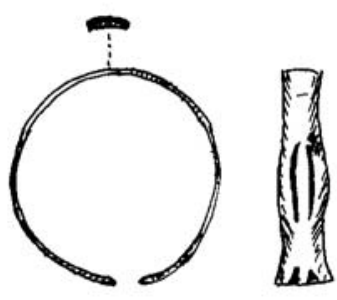
impressions niellées, autant sur les épaules de l'anneau, que sur sa partie supérieure, peut suggérer le motif des mains rassemblées, symbole des fiançailles. Pièce très détériorée.

Dm.ext. anneau: 20 mm; G.: 1,45 gr.

M.46: Anneau d'argent tordu et les extrémités entrelacées, reproduisant schématiquement la tête d'un serpent.

Dm.ext. anneau:

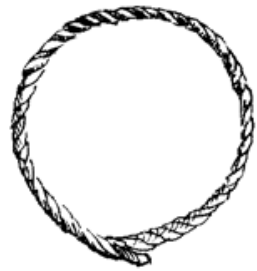


24,5 mm; G.: 1,45 gr.

M.47: Cinq boutons globulaires d'une feuille de métal commun, dorés à l'extérieure, ayant chacun à la
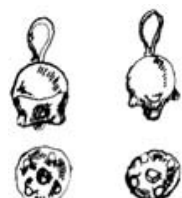
protubérances, le centrale étant plus grande. Dm.: $6 \mathrm{~mm}$; h.: $12 \mathrm{~mm}$.

M.48: Applique de métal commun, d'une forme ovale concave, un orifice central pour être fixer.

Dm.: $16 \mathrm{~mm}$ x $11 \mathrm{~mm}$.

M.51 Un bouton d'une feuille d'argent, d'une forme globulaire, fragmentaire.

M. 59: 1-2: Paire de boucles d'oreille d'argent à petite anse demi-circulaire, ayant à la partie inférieure trois petite roues granulées, soudées entre elles, flanquées par un fil tordu. L'un des bouts a une toute

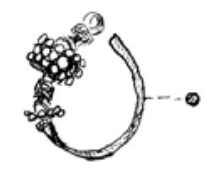
petite roue granulée, l'autre un anneau de fixation. A la deuxième boucle d'oreille manquent les petites roues centrales; celle terminale étant fragmentaire.

Dm.ext. anneau: $11 \mathrm{~mm}$; dm. La petite roue centrale: $6 \mathrm{~mm}$; G: 1 gr., respectivement 0,65 g.

3.: 14 boutons globulaires (deux fragmentaires) faits d'une feuille

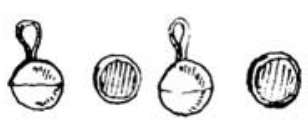
mince d'argent dorée, ayant à la partie supérieure un anneau de fixation.

Dm.: 6 mm; h.: 13 mm.

M. 60: 1. Anneau d'argent, tordu et visiblement plus gros à sa partie inférieure. Les extrémités sont tout proches. Pièce un peu déformée.

Dm.ext. anneau: 20

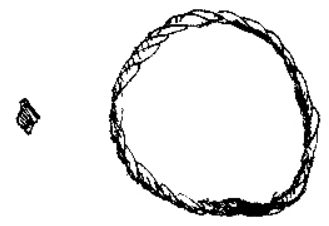
$\mathrm{mm}$; G.: 1,05 gr.

2. Fragment de petite perle tubulaire en verre.
M. 61: 1. Bague d'argent ayant l'anneau ovale en section, sur le quel a été soudé un chaton rectangulaire avec les coins un peu arrondis. Le décor réalisé par incision, contient dans le champ central une croix de

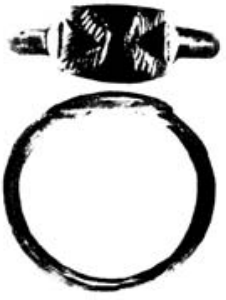
Malte, ayant l'espace d'entre les bras avec des registres en hachures.

Dm.ext. anneau: $24 \mathrm{~mm}$; monture: L.: $14 \mathrm{~mm}$; 1.: $7 \mathrm{~mm}$; G.: 3,60 gr.

\section{Fragment de bouton en bronze}

M. 62: Anneau d'argent tordu et les extrémités tout proches. Fortement déformé.

Dm.ext. anneau: 23

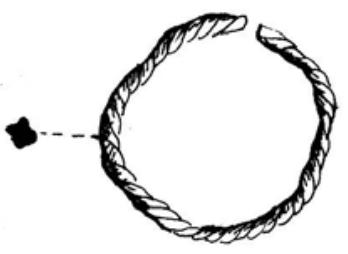
mm; G.: 1,65 gr.

M. 63: Bague d'argent ayant l'anneau demicirculaire en section et la partie inférieure fissurée. La monture, qui fait corps commun avec l'anneau, un peu surélevée d'une forme ovale, a encastrée une pierre demiprécieuse d'une couleur blanc-

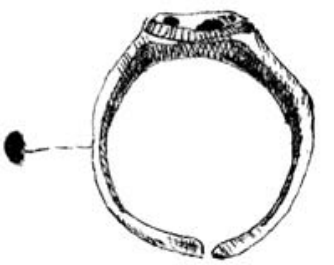
jaunâtre, probablement un agate. Le bord du chaton décoré avec un cadre d'incisions verticales.

Dm.ext. anneau: $22 \mathrm{~mm}$; dm. monture: 10 x 7 mm; G.: 3,22 gr.

M. 64: Bague d'argent ayant l'anneau d'un gros fil retors, sur le quel a été soudée une pastille rhom-
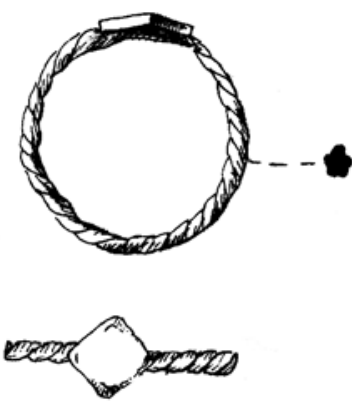
boïdale, non décorée.

Dm.ext. anneau: $23 \mathrm{~mm}$; côte du rhombe: 6 mm; G.: 2,80 gr.

M. 65: Fragment de petite perle en verre d'une couleur bleu-vert, ayant la section rectangulaire.

M. 69: Anneau d'argent d'un fil retors. Les extrémités étant plus minces et superposées.

Dm.ext. anneau: 20

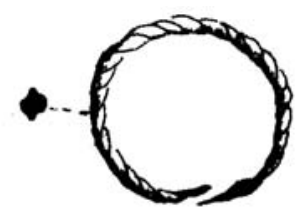
mm; G.: 1,05 gr.

M. 72: 1. Deux petites perles d'une pâte vitreuse, carrée en section, une plus grande de couleur bleu vert, l'autre plus petite d'une couleur grise.

2. Deux boutons fragmentaires globulaires, faits d'une feuille mince de métal commun.

M. 73: 1. Quatre perles d'une pâte vitreuse, extrêmement corrodées. Trois sont circulaires, d'une couleur bleue, une tubulaire de couleur bleue et une perle en terre cuite ayant les rebords lobés en forme de fleure à six pétales.

2. Boutons fragmentaires en métal commun.

M. 74: Trois boutons fragmentaires globulaires, obtenus de deux hémisphères soudées d'une feuille mince de métal commun.

M. 75: Deux boutons fragmentaires globulaires, réalisés deux hémisphères soudées d'une feuille mince de métal commun.

M. 76: 1. Boucle d'oreille fragmentaire, de la quelle s'est gardé seulement la partie centrale, réalisée par l'entrelacement de trois fils d'argent tordu en forme de bouton de fleur. L'anneau fragmentaire réalisé

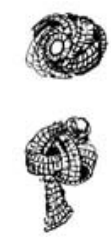
de même d'un fil retors d'argent.

Dm.: 8 mm; G.: 1,10 gr.
2.: Bouton globulaire, réalisé de deux hémisphères soudées d'une feuille mince d'argent et ayant un anneau de fixage.

Dm.: $5 \mathrm{~mm}$; h.: 10,5 mm.

M. 98: 1. Bague d'argent dorée ayant l'anneau demicirculaire en section et qui fait corps commun avec la monture un peu surélevée, d'une forme octogonale, décorée avec un motif géométrique incisé (probablement un motif zodiacal - poisson). Les

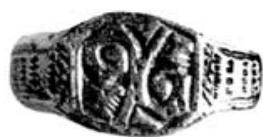
épaules de l'anneau sont gravées avec des registres de lignes verticales, et à la partie inférieure de l'anneau, une bande de lignes incisées.

Dm.ext. anneau: $22 \mathrm{~mm}$; dm. monture: 10 x 9,8 $\mathrm{mm}$; G.: 8,60 gr.

2. petite perle tubulaire en verre de couleur bleue nacrée.

M. 99: Anneau en bronze, circulaire en section et les extrémités tout proches.

Dm.ext. anneau: $22 \mathrm{~mm}$.

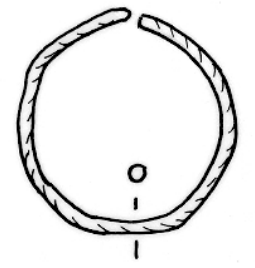

M. 100: Neuf boutons d'une forme globulaire (un fragmentaire), réalisés de deux hémisphères soudés d'une feuille mince de métal commun, dorées à l'extérieure, le fond biseauté et un anneau de fixage.

Dm.e: $6 \mathrm{~mm}$;h.: $9 \mathrm{~mm}$.

M. 103: Fragment de petites perles en verre d'une section carré, ayant la couleur blanche nacrée.
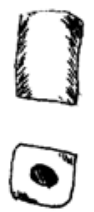
M. 125: Bagues d'argent ayant l'anneau d'un fil circulaire en section et avec des incisions sur le rebord extérieure, ayant comme monture une pastille circulaireovale soudée, son décor reproduisant une fleure à quatre pétales.

Dm.ext. anneau: 22 $\mathrm{mm}$; dm. monture: 6 mm; G.: 2,45 gr.

M. 126: Bague d'argent ayant l'anneau d'un fil retors, avec les épaules très minces et une pastille soudée, d'une forme ovale à décor indéchiffrable.

Dm.ext. anneau: $22 \mathrm{~mm}$; dm. monture: $6 \mathrm{~mm}$; G. 1,95 gr.

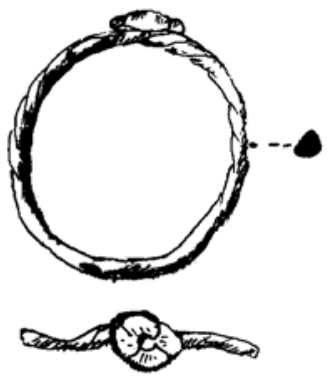

M. 130: Petite perle en verre de couleur bleue jaunâtre, carrée en section.

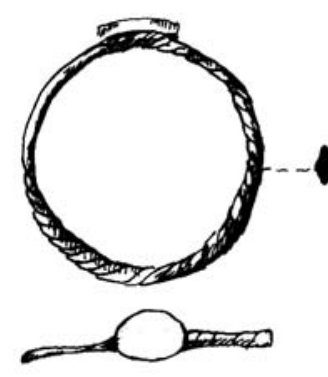

couleur bleu nacrée.

M. 207: Enfilade de 11 perles, carrées et rectangulaires en section, de différentes dimensions, d'une pâte vitreuse de couleur bleu foncé et blanche.
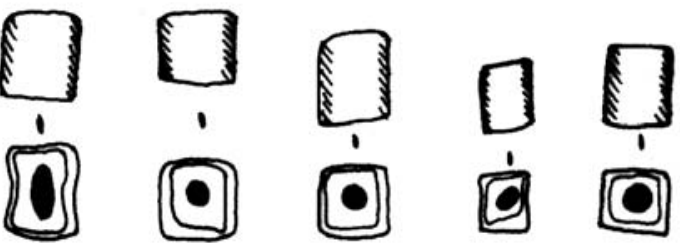

M. 208: Trois perles en verre, de

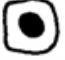

M. 233: Bague d'argent ayant l'anneau de fil retors, avec les extrémités soudées. La monture est faite d'une pastille rhombique soudée et elle est décorée avec le motif de la croix (de Malte?) incisé dans un cadre en relief.

Dm.ext. anneau: $27 \mathrm{~mm}$; dm. monture: 7 x 6 mm; G.:

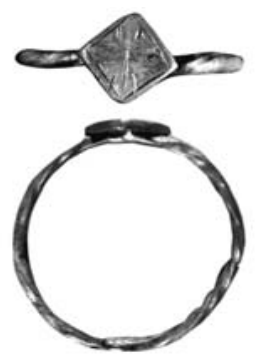
G.: 0,95 gr. 2,68 gr.

M. 158: Petit fragment de tissue en laine.

M. 160: Fragment de bouton globulaire en bronze.

M. 202: 1. Bague d'argent ayant l'anneau en fil retors, avec la monture d'une pastille rhombique soudée, qui a comme décor un motif florifère (trèfle à quatre feuilles ?) disposé dans un cadre de points incisés.

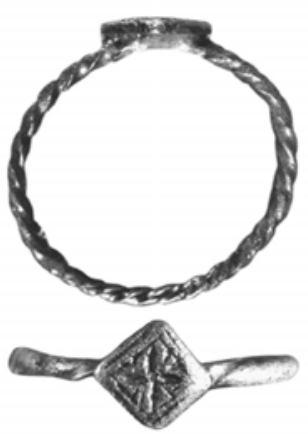

M. 246: 1.

Boucle d'oreille fragmentaire de feuille en métal commun, dorée à l'extérieure, et d'une forme sphéroïdale,
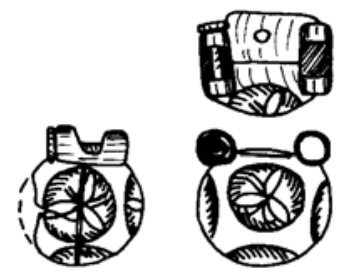
ayant à sa partie supérieure deux tubes cylindriques par les quels passait l'anneau, et le corps décoré en gravure avec cinq registres ornementés de rosettes.

Dm.ext.: 16 mm; h.: 14 mm. 
2. Collier de perles contenant 18 exemplaires de divers dimensions, circulaires ou carrées, d'une pâte vitreuse, des couleurs: blanc, bleu ouvert et bleu foncé, vert. Quatre exemplaires ont
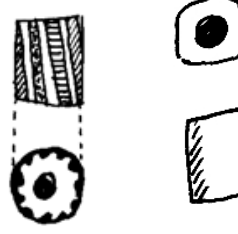
peintes des lignes horizontales vertes, rouges et bleus, sur un fond gris vert.

M. 248: Bague d'argent ayant l'anneau demicirculaire et les épaules biseautés faisant corps commun

avec la

monture.

Celle-ci,

d'une

forme

ovale plate

est décorée

avec un
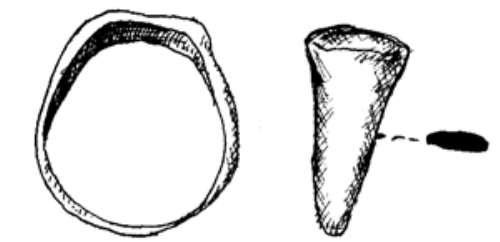

motif

zoomorphe: deux oiseaux incisés, en profil vers la gauche.

Dm.ext. anneau: $20 \mathrm{~mm}$; dm. monture: $11,5 \mathrm{x}$ $11,3 \mathrm{~mm}$; G.: 7,20 gr.

M. 253: Quatre boutons globulaires (deux fragmentaires), obtenus de deux hémisphères soudées d'une feuille mince de métal commun, argentée à l'extérieur.

M. 271: Boucle d'oreille fragmentaire de métal commun. S'est gardé seulement la petite anse circulaire en section, ayant une des

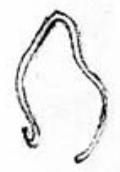
extrémités avec un anneau de fixage.

h.: $15 \mathrm{~mm}$

M. 275: Petite anse d'un bouton en bronze.

M. 282: Anneau d'argent, d'un fil simple, rond en section, avec les extrémités entrelacées.

Dm.ext. anneau: 20,5 mm;

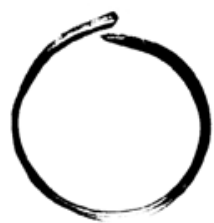
G.: 0,65 gr.
M. 301: Petite perle d'une pâte vitreuse, circulaire en section.

M. 310: 1. Bague d'argent, ayant l'anneau demi-circulaire en section, qui fait corps commun avec la monture. Les épaules de l'anneau sont gravées avec des motifs végétaux (fleure de lys), par ci par la, des traces de niellage. Le chaton d'une forme ovale, un peu surélevé présente sur le rebord extérieure un registre niellé, entouré par deux cadres d'incisions obliques. La plaque du chaton est décorée avec le motif de l'aigle bicéphale, vue de face, avec les ailles et la queue défaites, les têtes en profil, tout bordé d'un registre de lignes incisées.

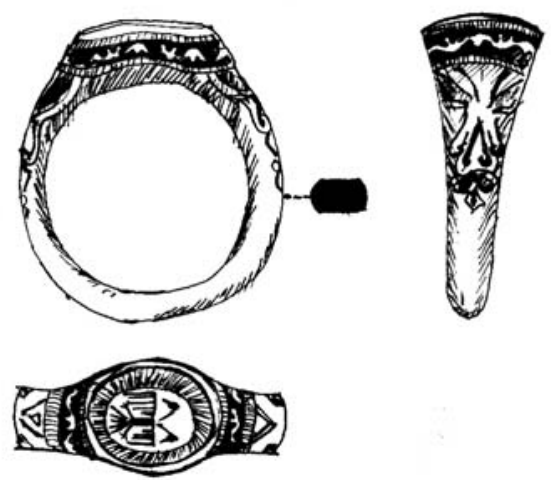

Dm.ext. anneau: $24 \mathrm{~mm}$; dm. monture: $14 \mathrm{x} 11$ $\mathrm{mm}$; G.: 7,20 gr.

2. Cinq boutons (un fragmentaire) globulaires, réalisés de deux hémisphères

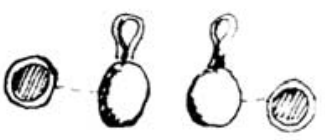
soudées d'une feuille mince d'argent.

Dm.: $5 \mathrm{~mm}$; h.: $11 \mathrm{~mm}$.

M. 313: 1. Paire de boucles d'oreille en bronze avect l'anse circulaire, sur elles étant fixé par un bouton
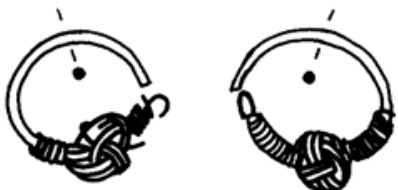
circulaire obtenu à l'aide de l'entrela-cement de trois fils de bronze. Le bouton est flanqué des deux cotés 
par un fil retors; une boucle d'oreilles est un peu déformée.

Dm. anneau: $15 \mathrm{~mm}$; dm. Bouton $5 \mathrm{~mm}$.

2. Six boutons (un fragmentaire) globulaires, obtenus de deux hémisphères soudées d'une feuille mince de métal commun, biseautés à leur partie inférieure et ayant un anneau de fixage.

Dm.: 6 mm; h.: 9 mm.

M. 327: 1. Bague d'argent ayant l'anneau demi-circulaire, qui fait corps commun avec la monture un peu plate, ornementée par incisions avec un motif solaire, qui suggère un petit moulin. Les épaules décorées en trois registres de points incisés. $\mathrm{La}$ partie inférieure de l'anneau contient un bouton.

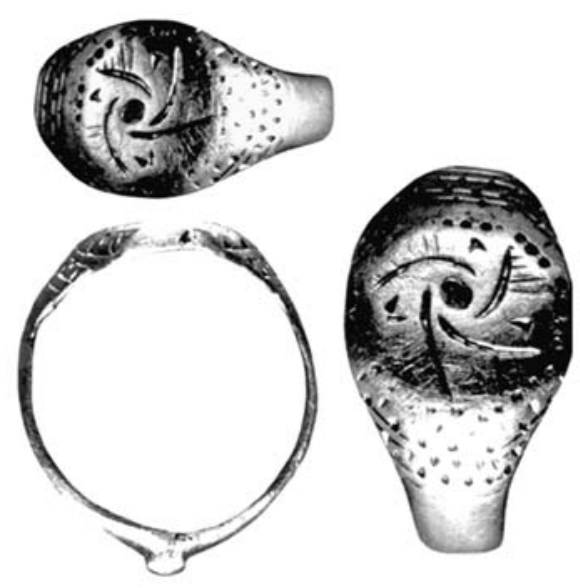

Dm.ext. anneau: $20 \mathrm{~mm}$; dm. monture: $10 \mathrm{~mm}$; G.: 5,35 gr.

2. Deux boutons (fragmentaires) globulaires, obtenus de deux hémisphères soudées d'une feuille de métal commun; seulement les anneaux de fixage et quelques hémisphères.

M. 335:

Bague

d'argent,

ayant

l'anneau

demi-

circulaire, à

la partie

inférieure un

bouton fort

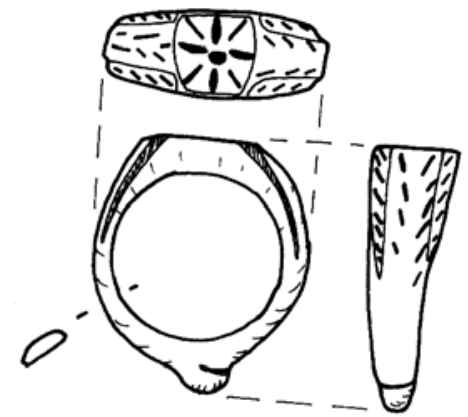

en relief. Les épaules ont un profil rectangulaire arrondi, dé-coré par trois cadres de points incisés. La mon-ture, qui fait corps com-mun avec l'anneau, un peu plat, est décorée avec un motif solaire incisé.

Dm.ext. anneau 21,5 mm; dm. monture: $11 \times 10$ mm; G.: 8,60 gr.

M. 341: Bague d'argent, ayant l'anneau demicirculaire, la monture ovale, faisant corps commun

l'anneau. avec monture décorée par incisions avec un motif zoomorphe indéchiffrable en profil (chèvre). Chaque épaule a le

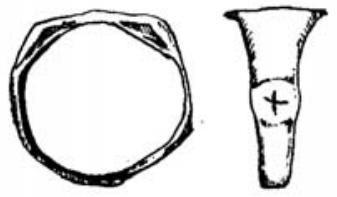
profil rectan-gulaire arrondi. La pièce est très usée.

Dm.ext. anneau: $21 \mathrm{~mm}$; dm. monture: $12 \times 9$ mm; G.: 10,90 gr.

M. 344: Bague d'argent ayant l'anneau d'un fil retors, avec une petite pastille ovale soudée, non décorée.

Dm.ext. anneau: $22 \mathrm{~mm}$; $\mathrm{dm}$. monture: $5 \mathrm{~mm}$; G.:

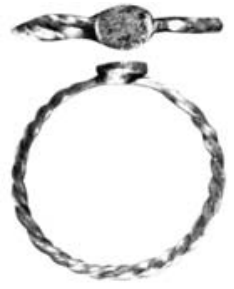
$1,52 \mathrm{gr}$.

M. 346: 1. Anneau d'un fil mince d'argint avec les extremités tout proches.

Dm.ext. anneau: $16 \mathrm{~mm}$; G.: 0,55 gr.

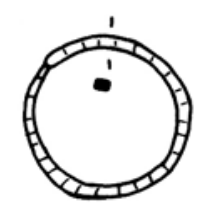

\section{Bouton fragmentaire. Seulement l'anneau}

M. 349: Boucle d'oreille fragmentaire, très déformée, en argent, l'anneau demi-circulaire, et un petit orifice de fixage, décorée avec des minces incisions à la partie inférieure et

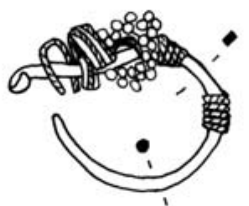


avec des petites roues granulées mises en pyramide et des fils d'argent tordus.

Dm.: 16 mm; G.: 1,63 gr.

M. 352: Deux fragments d'anneaux d'argent (?) d'une boucle d'oreille.

M. 360: 1. Anneau d'argent d'un fil retors et les extrémités plates et tout proches.

Dm.ext. anneau: 21,5 mm; G.: 1,20 gr.

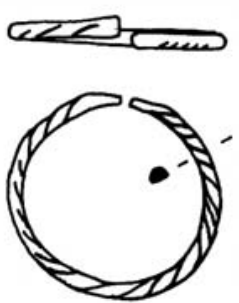

2. Trois boutons (un d'argent, deux de métal commun) globulaires, faits d'une seule feuille mince, ayant la partie inférieure biseautée.

Dm.: $6 \mathrm{~mm}$; h.:11 mm; Gr.: 0,38 gr. (celui d'argent)

M. 379: Bouton globulaire, obtenu d'une seule feuille mince d'argent, ayant un anneau de fixage.

Dm.: 7 mm; h.: 10 mm; Gr. 0,33 gr.

M. 381: 1. Anneau d'argent d'un fil retors et les extrémités tout proches. La pièce est un peu déformée.

Dm.ext. anneau: $22 \mathrm{~mm}$; G.: 0,97 gr.

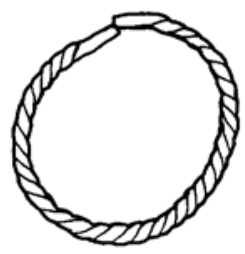

2. Deux boutons globulaires, fragmentaires, en métal commun.

Dm.: $6 \mathrm{~mm}$; h.: $11 \mathrm{~mm}$.

M. 408: 1. Anneau d'argent d'un fil retors et les extrémités superposées.

Dm.ext. anneau: 22 mm; G.: 1,15 gr.
2. Six boutons globulaires, trois fragmentaires, trois dorés à l'exté-rieure, d'une feuille mince, contenant un anneau de fixage et 4 protubérances à la partie inférieure, celle centrale plus en relief.

Dm.: $7 \mathrm{~mm}$; h.:12 mm.

M. 421: 1. Anneau d'argent d'un fil simple avec les extrémités tout proches.

Dm.ext. anneau: $21 \mathrm{~mm}$;

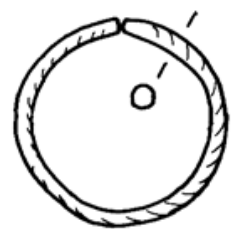
G.: 0,98 gr.

2. Trois boutons globulaires, obtenus d'une feuille mince de métal commun, ayant l'anneau de fixage et la partie inférieure biseautée.

Dm.: $6 \mathrm{~mm}$; h.:11 mm.

M. 425: Petite perle en pâte vitreuse de couleur noire, d'une forme bitronconique, décorée avec des incisions de couleur

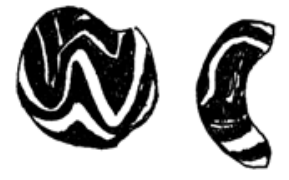
blanche, qui suggères les nervures d'une feuille. L.: $30 \mathrm{~mm}$.

M. 445: Anneau en métal commun, demicirculaire en section, qui fait corps commun avec la monture, d'une forme circulaire et non décoré (pièce non illustrée, en cours de restauration). 


\section{Pièces sans contexte funéraire}

1. Anneau en bronze, provenant de la Section XVI/ 1991, $\square 5,-0,40 \mathrm{~m}$.

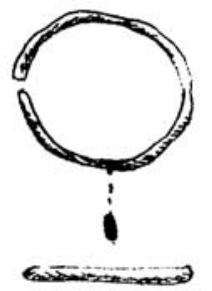

2. Fragment de boucle d'oreille en bronze, ornementé avec des petites roues granulées et des fils retors provenant de la Section XXXII/ 1992, $\square$ 12, - 0,20 m.
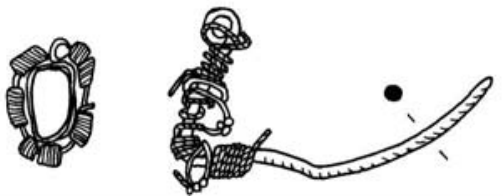

\section{BIBLIOGRAPHIE}

Chițescu ş. a. 1979 - L. Chițescu, N. Conovici, R. Lungu, A. Păunescu, V. Rădulescu, Cercetări arheologice de la Piua Petri (Oraşul de Floci), jud. Ialomița. Cercetări Arheologice 3, 1979, 199 - 244.

Chițescu ş. a. 1981 - L. Chițescu, R. Lungu, T. Papasima, P. Vlădilă, V. Rădulescu, A. Păunescu, Cercetări arheologice în anul 1979 la Piua Petri (Oraşul de Floci), comuna Giurgeni, jud. Ialomița. Cercetări Arheologice IV, 1981, 120 - 143.

Chițescu ş. a. 1982 - L. Chițescu, T. Papasima, P. Vlădilă, V. Rădulescu, A. Păunescu, Cercetări arheologice de la Piua Petri (Oraşul de Floci), jud. Ialomița. Cercetări Arheologice V, 1982, 129 - 158.

Dumitriu 2001 - L. Dumitriu, Der mittelalterliche Schmuck des unteren Donaugebietes in 11. - 15. Jahrhundert. Bucurest 2001.

Panait, Ştefănescu 1992 - I. P. Panait I. P., A. Ştefănescu, Situl Bârzeşti. Cercetări Arheologice în Bucureşti IV, 1992, $147-160$.

Rosetti 1972 - Rosetti D. V., Vestigiile feudale de la Suslăneşti, jud. Argeş. Buletinul Monumentelor Istorice şi de Artă, 41, 2, 1972, 27-39.

Ştefănescu 1979 - Ştefănescu A., Cercetări arheologice în raza oraşului Buftea. Revista Muzeelor 3, 1979, 22-30.

Stefănescu 1980 - Ştefănescu A., Cercetarea arheologică a satului din Țara Românească (sec. XIV-XV). Studii şi Cercetări de Istorie Veche, 21, 1, 1980, 131-141.

\section{Verzeichnis der Abbildungen}

Abb. 1. Tabelle der Schmuck- und Trachtstücke aus dem Gräberfeld von Vadu Anei.

Taf. 1. Vadu Anei. Dei Typologie der Ohrringe

Taf. 2. Vadu Anei. Dei Typologie der Fingerringe (die Typen $3.1 \mathrm{a}-\mathrm{b}$ ).

Taf. 3. Vadu Anei. Dei Typologie der Fingerringe (die Typen $3.2 \mathrm{a}-\mathrm{b}$ ).

Taf. 4. Vadu Anei. Dei Typologie der Fingerringe (die Typen $3.3 \mathrm{a}-\mathrm{b}$ ).

Taf. 5. Vadu Anei. Die Typologie der Knöpfe (die Typen $4.1 \mathrm{a}-\mathrm{b}$ ).

Taf. 6. Vadu Anei. Fingerringe (M310, M335, M61, M63, M98, M327, M248, M341).

Taf. 7. Vadu Anei. Fingerringe (M3, M126, M125, M344, M233, M11, M43, M64, M202, M45, M421, M282).

Taf. 8. Vadu Anei. Schmuck stüke aus dem gräberfeld von Vadu Anei (M59, M313, M76, M246, M425).

\section{Luminița Dumitriu}

Muzeul Naţional de Istorie a României

Calea Victoriei 12, RO-030026, București 